\title{
Procedimientos intervencionales guiados por ultrasonido en radiología musculoesquelética
}

\author{
Drs. Julio Rosales $L^{(1)}$, Sebastián Butrón $M^{(1,2)}$, Christian Linderman $R^{(1)}$, Marcos Verdugo ${ }^{(1)}$, Giancarlo Schiappacasse $F^{(3)}$, \\ Roberto Yañez $D^{(4)}$.
}

1. Servicio de Imágenes, Centro Médico MEDS, Santiago, Chile.

2. Departamento de Radiología, Hospital Militar, Santiago, Chile.

3. Facultad de Medicina Clínica Alemana - Universidad del Desarrollo. Santiago, Chile.

4. Servicio de Traumatología y Ortopedia, Centro Médico MEDS, Santiago, Chile.

\section{Ultrasound guided interventional procedures in musculoskeletal radiology}

Abstract: Ultrasound is a useful diagnostic modality to study many structures such as subcutaneous tissue, tendons, muscles, joints, and nerves. It has low cost, wide availability and high resolution. These advantages make ultrasound a good modality in interventional procedures like soft tissue tumors biopsy, aspiration of cysts and other collections, and also in treating symptomatic calcifications like deposition of hidroxiapatite crystals in the rotator cuff, among other indications. Our objetive is to present the experience of the authors in performing musculoskeletal interventional procedures by ultrasound.

Keywords: Interventional procedures, Musculoskeletal, Ultrasound.

Resumen: El ultrasonido es una modalidad imaginológica útil para el estudio de múltiples estructuras, tales como tejidos subcutáneos, tendones, músculos, articulaciones y nervios. Tiene un bajo costo, amplia disponibilidad y alta resolución. Estas ventajas hacen del ultrasonido una excelente modalidad en procedimientos intervencionales tales como biopsias de tejidos de partes blandas, aspiración de quistes y otras colecciones y también en el tratamiento de calcificaciones sintomáticas, tales como depósitos de cristales de hidroxiapatita de calcio en el manguito rotador. Este artículo desea mostrar la experiencia de los autores en la realización de procedimientos intervencionales musculoesqueléticos guiados por ultrasonido. Palabras clave: Musculoesquelético, Procedimientos Intervencionales, Ultrasonido.

Rosales $\boldsymbol{J}$ y cols. Procedimientos intervencionales guiados por ultrasonido en radiología musculoesquelética. Rev Chil Radiol 2011; 17(4): 159-163.

Correspondencia: Dr. Julio Rosales L. / jurosle@ hotmail.com

Trabajo recibido el 24 de octubre de 2011, aceptado para publicación el 12 de diciembre de 2011.

\section{Introducción}

Los múltiples métodos de examen en la práctica de la imaginología, ya no sólo se limitan al estudio diagnóstico de las diversas patologías, sino que también al apoyo de su tratamiento.

Dentro de ellas, el ultrasonido ha experimentado un progresivo aumento en su utilización en el área musculoesquelética como guía para procedimientos de diversa índole y con mínima invasividad, siendo muy bien tolerados y aceptados por el paciente, además de producir escasas complicaciones cuando son realizadas por profesionales entrenados.

De las varias características de esta modalidad, destacan su capacidad de imagen en tiempo real, multiplanaridad, alta resolución espacial, ausencia de radiación ionizante, bajo costo y amplia disponibilidad. Es fundamental contar con transductores lineales de alta resolución de al menos $12-5 \mathrm{MHz}$, que facilitan una adecuada visualización y diferenciación de las estructuras del sistema musculoesquelético ${ }^{(1,2)}$.

Adicionalmente, la función Doppler color es de utilidad, ya que permite reconocer el paquete vásculo-nervioso vecino a la zona a puncionar, y de esta manera, evitar que sea lesionado durante el procedimiento.

El objetivo de esta publicación, es la de presentar la experiencia adquirida en los últimos 5 años en intervención guiada por ultrasonido, para el tratamiento y manejo de una amplia variedad de patologías del área musculoesquelética.

Entre éstas podemos señalar las tendinopatías, lesiones musculares y ligamentosas (especialmente aquellas ligadas a la práctica deportiva), procesos inflamatorios articulares, tratamiento de patología bursal y lesiones quísticas. Conforme aumenta la experiencia y mejoran los resultados, se incrementa 
la aceptación por parte de los médicos clínicos y se suman nuevas indicaciones.

\section{Técnica}

Para la realización de los procedimientos intervencionales se requiere del equipamiento ecográfico adecuado, conocimiento acabado de la anatomía regional y de un médico radiólogo calificado y con alto entrenamiento en el uso del ultrasonido en el estudio de la patología a tratar. Dichas intervenciones se realizan con técnica estéril, material desechable y utilizando accesos que resulten lo menos traumáticos para los pacientes ${ }^{(1,2)}$. Todo procedimiento médico realizado por un radiólogo debe cumplir una serie de pasos con el fin de maximizar las posibilidades de éxito y minimizar los riesgos. Por ello, en nuestra institución, se cumple con un estricto protocolo que a continuación se describe.

1. Dar una explicación detallada al paciente del procedimiento y de las eventuales complicaciones que puede sufrir luego de éste. Posteriormente, se procede a entregar una solicitud de consentimiento escrita y que debe ser firmada por el paciente u otra persona responsable en el caso que éste estuviera impedido(3).

2. Repetir el examen ecográfico en la zona corporal afectada con el propósito de confirmar la patología diagnosticada y elegir la vía de acceso más adecuada para la realización de la punción. Dicha vía es aquella que implique tanto la distancia más corta entre la piel y la lesión, así como la posición más cómoda tanto para el paciente como para el operador.

3. Recostar al paciente para que se mantenga cómodo, relajado y exponiendo adecuadamente el segmento corporal a ser tratado. Rara vez se realizan estos procedimientos con el paciente sentado.

4. El radiólogo debe realizar lavado de manos y a continuación utilizar guantes estériles y mascarilla.

5. Practicar un aseo amplio de la piel y posteriormente aplicar un antiséptico local. Con paños estériles se delimita un amplio campo de trabajo.

6. Preparar el material estéril con el que se va a llevar a cabo el procedimiento. La elección de éstos depende de la intervención, pero comprende al menos una serie de agujas, jeringas, fármacos (por ejemplo soluciones corticoidales), anestésico local y gel ecográfico estéril (Figura 1).

7. Se cubre el transductor del ecógrafo con un cobertor estéril.

8. Dependiendo si el procedimiento consiste en la infiltración y/o aspiración de fluidos se debe considerar el siguiente material básico:

a) Agujas: para infiltraciones de quistes simples, se recomienda utilizar agujas con calibres mayores (18 o 19G). En cuanto a la profundidad, para las lesiones más superficiales se utilizan agujas con longitudes de 25 a $38 \mathrm{~mm}$ y para lesiones profundas se debe utilizar agujas espinales de 7 a $10 \mathrm{~cm}$ de largo.

b) Corticoides: se prefiere la administración de soluciones corticoidales de largo efecto en volúmenes de 1 a $2 \mathrm{cc}$, habitualmente asociado a pequeña cantidad de anestésico local. Se debe advertir al paciente la posibilidad de desarrollar un cuadro inflamatorio doloroso regional autolimitado, post-punción, correspondiente a una sinovitis por cristales, la cual se trata con frío local y antiinflamatorios no esteroidales. Ante otras eventualidades, se recomienda al paciente comunicarlas de inmediato al equipo tratante.

9. Se utiliza preferentemente la técnica de "mano libre", en la cual con una mano se controla el transductor y con la otra se introduce la aguja. Previa decisión de la vía de acceso adecuada y realizando la menor cantidad de punciones posibles, la aguja debe ingresar en forma longitudinal al eje largo del transductor y su punta debe ser visualizada permanentemente durante el procedimiento direccionándola hacia el objetivo ${ }^{(4)}$.

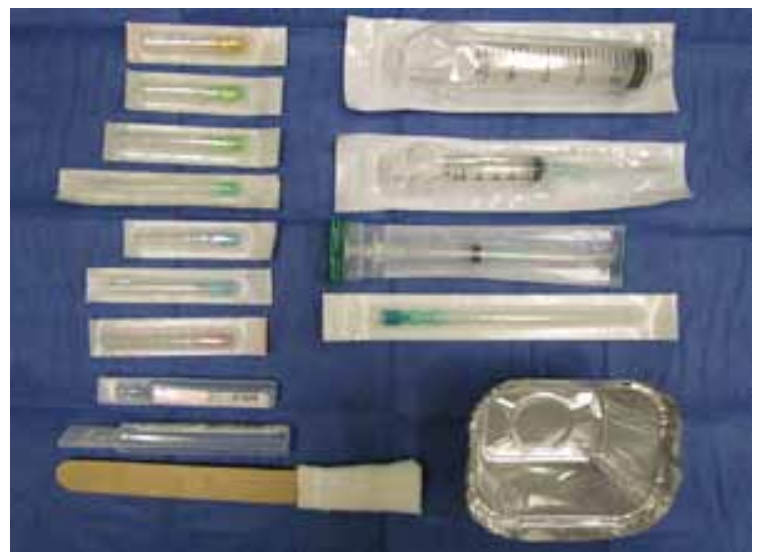

Figura 1. Material básico de trabajo dispuesto sobre bandeja.

Un detalle más preciso de cada técnica realizada en los diversos procedimientos intervencionales será motivo de futuras publicaciones.

Debemos destacar, que toda decisión o indicación acerca de los procedimientos realizados por nosotros, proceden del análisis clínico previo realizado entre el médico tratante, ya sea traumatólogo, reumatólogo o fisiatra y el radiólogo responsable.

De los procedimientos realizados más frecuentemente en la práctica diaria se puede mencionar:

\section{Punción evacuadora de gangliones y quistes sinoviales}

Los quistes gangliones y sinoviales son la causa de aumento de volumen de partes blandas más común, especialmente en muñecas, pero también en otras localizaciones menos frecuentes, como por ejemplo, hueco poplíteo, tobillo y articulación 
acromio-clavicular(4). En nuestra experiencia (no publicada), cerca de dos tercios de las localizaciones corresponden a muñeca y mano, un tercio a rodilla y tobillo y muy raramente otros sitios.

Muchos de ellos son asintomáticos, por lo que no requieren tratamiento. Reportes señalan regresión espontánea en un 40 a $60 \%$ de los casos $^{(3)}$.

Se efectúa tratamiento en aquellos que no regresan y que generan síntomas, habitualmente dolor y efecto compresivo. La cirugía tiene la tasa más alta de éxito, alcanzando más de un $95 \%$ de efectividad, sin embargo, las complicaciones pueden ser mayores ${ }^{(5)}$.

La aspiración mediante punción guiada por ecografía es una buena alternativa dado que es una técnica mínimamente invasiva, de alta efectividad y con bajas complicaciones ${ }^{(4,5)}$. Posterior al procedimiento de drenaje, se complementa con la inyección de un fármaco corticoidal intralesional con el objetivo de disminuir la posibilidad de recurrencia ${ }^{(4,6)}$ (Figura 2).

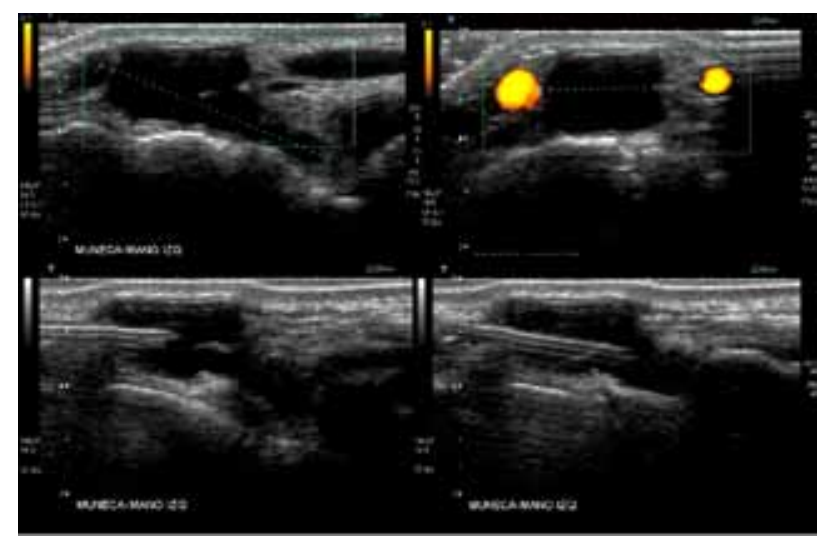

Figura 2. Quiste sinovial de la muñeca: Punción y aspiración de quiste de la muñeca guiado por us. Con función doppler color se detectan los vasos adyacentes con el objetivo de evitar dañarlos.

También esta técnica es aplicable a otras localizaciones, tales como quistes paralabrales de hombro y cadera $^{(7)}$.

\section{Bursitis}

El proceso inflamatorio bursal no séptico, es una entidad común en la práctica deportiva, siendo la más frecuente en nuestra actividad la subacromiosubdeltoidea. El empleo de corticoides y anestésicos locales, permite aliviar el dolor producido por esta patología (Figura 3). La dosis y tipo de fármaco a utilizar depende, tanto de la cuantía del proceso como de su disponibilidad o preferencia por parte del operador $y / 0$ tratante ${ }^{(4,8,9)}$. Se utiliza la técnica de punción antes descrita, con el paciente posicionado en decúbito dorsal y con la extremidad superior dispuesta paralela al eje longitudinal del cuerpo o bien con el antebrazo descansando sobre el abdomen dependiendo de aquella con la que mejor se obtenga una visualización de la bursa.

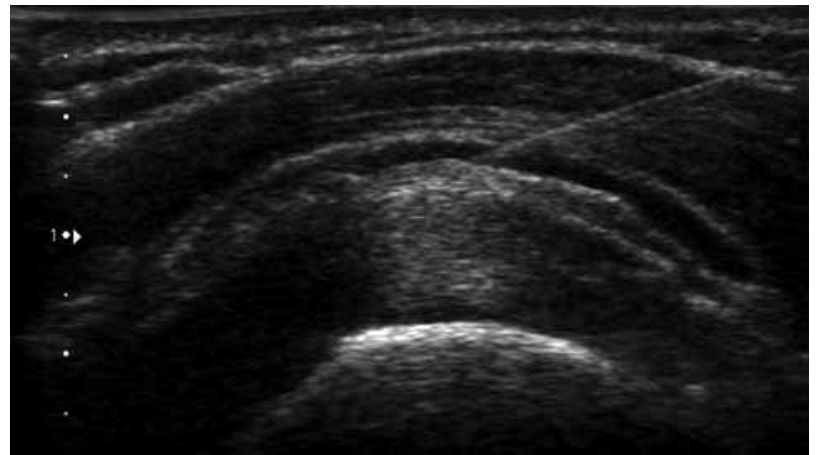

Figura 3. Bursitis subacromial subdeltoidea: Con guía ultrasonográfica se realiza una punción intrabursal e infiltración de solución corticoidal. Mediante esta técnica, es posible utilizar una dosis mínima efectiva, evitando además punción intratendínea y/o extrabursal.

\section{Aspiración de calcificaciones del manguito rotador}

El ultrasonido es muy sensible en la identificación de calcificaciones intratendíneas. Bajo visión ecográfica directa y con técnica de una aguja, se efectúa punción de la calcificación con una aguja gruesa (18G), lavado con suero fisiológico y lidocaína; posteriormente se practica su aspiración (Figura 4). Inicialmente se obtiene un lavado "lechoso". Se practica este procedimiento hasta que el líquido obtenido es cristalino. En los casos en que la calcificación sea muy compacta y no permita su lavado, se procede a su fragmentación, tanto como sea posible, procurando no dañar el tendón aledaño, con lo que el contenido cálcico se liberará a la cavidad bursal adyacente desde donde se reabsorberá espontáneamente. Calcificaciones residuales en el tendón pueden sufrir la misma evolución por lo que su persistencia no debe considerarse como fracaso del tratamiento. Además, durante el procedimiento, se puede infiltrar la bursa subacromio-subdeltoídea con corticoide de depósito. La mejoría clínica es considerable, logrando un alivio significativo de la sintomatología en casi un $90 \%$ de los $\operatorname{casos}^{(10-12)}$.

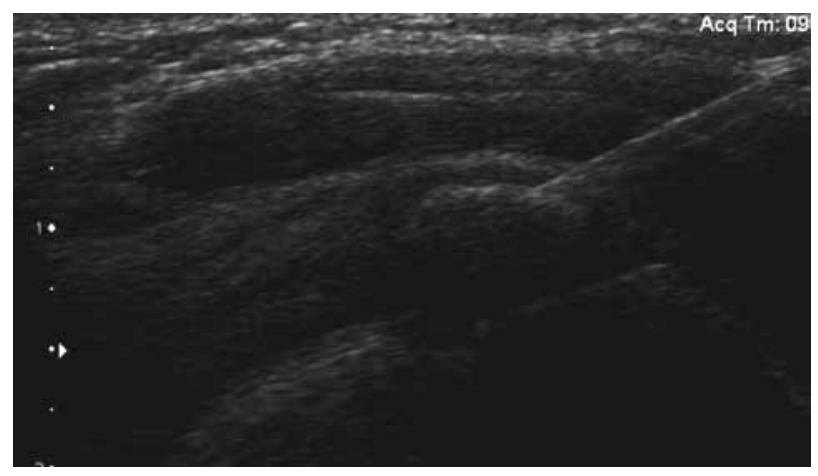

Figura 4. Tendinopatía cálcica del manguito rotador: Gruesa calcificación intratendínea del supraespinoso. Se efectúa punción y posterior aspiración. 


\section{Patología tendinosa}

Dentro de la amplia gama de lesiones tendíneas, la radiología tiene un rol terapéutico acotado al tratamiento de aquellas de la esfera de las tendinosis y pequeñas roturas intrasustancia ${ }^{(13)}$.

En la fisiopatología de las tendinosis se observan tres fases: inflamatoria, proliferativa y de remodelación. Durante la primera fase se pueden realizar procedimientos con el fin de abreviar dicho período, mediante el uso de infiltraciones peritendíneas de esteroides y lidocaína ${ }^{(13,14)}$. En las siguientes fases se está incrementando la indicación del uso de plasma rico en plaquetas (PRP) con la intención de favorecer la regresión y reparación de dichas lesiones (Figura 5) ${ }^{(15)}$. Entre las patologías más frecuentemente tratadas están las tendinopatías rotuliana y aquiliana ${ }^{(15)}$.
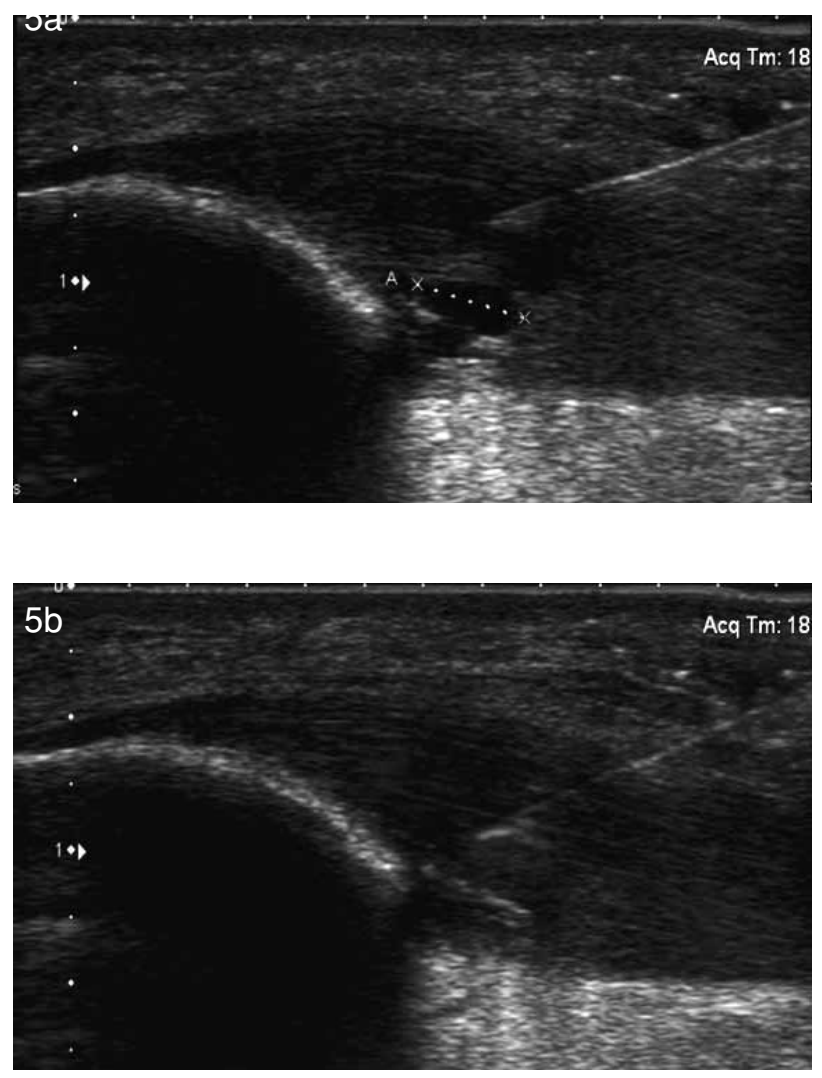

Figura 5. Paciente con tendinopatia rotuliana proximal y rotura fibrilar en la rodilla izquierda: Se procede a infiltración de prp intratendíneo.

El PRP consiste en un tipo de concentrado plaquetario que contiene, al menos, cinco veces la concentración sérica habitual de plaquetas, que en promedio es de 200.000 plaquetas $\times \mu \mathrm{L}$ (rango de 150.000 a $350.000 \times \mu \mathrm{L}$ ). Esta alta concentración de plaquetas también determina un aumento de la concentración de una serie de factores de crecimiento bioactivos que favorecerían los procesos de curación de las heridas ${ }^{(16)}$. Este se obtiene a través de una punción venosa al paciente, en la que se recolectan unos $30 \mathrm{cc}$ de sangre completa, asociada a un anticoagulante, la cual, luego de ser sometida a un proceso de centrifugado, se logra estratificar en tres capas distintas los diferentes componentes sanguíneos. Aquella más superficial consiste en plasma pobre en plaquetas (PPP), un segundo que está compuesto por PRP y leucocitos y una tercera capa, de disposición, sedimentaria que corresponde mayoritariamente a glóbulos rojos. El volumen de PRP es aproximadamente un 10\% del volumen inicialmente recolectado.

A la técnica de punción descrita, debe destacarse el hecho que no debe utilizarse anestésico intratendíneo debido a que inactiva el PRP. En estos procedimientos se pueden utilizar agujas de calibre intermedio o fino. EI PRP es distribuido en la rotura y en el tendón adyacente, a través de múltiples fenestraciones. Este procedimiento puede provocar intenso dolor en alguno pacientes, temprana o tardíamente, recomendándose el uso de analgésicos para su tratamiento y evitando la administración de antiinflamatorios no esteroidales puesto que pueden inactivar su función ${ }^{(16)}$. Idealmente, se recomienda suspender la administración de estos fármacos por dos semanas, tanto antes como después de este tratamiento.

\section{Lesiones musculares}

Estas se producen por contusión directa o bien por sobredistensión. El resultado final es la disrupción de fibras musculares y la formación de hematomas locales de variado tamaño. Mediante el intervencionismo guiado por ecografía es posible aspirar aquellos hematomas significativos con la consiguiente mejoría de síntomas y, al mismo tiempo, administrar $\mathrm{PRP}^{(15)}$, con lo que se pretende por un lado, acelerar el tiempo de recuperación, y por otro, disminuir el exceso de fibrosis (Figura $6^{(16)}$. Se ha descrito una disminución en el tiempo de recuperación de hasta un $50 \%$, en comparación con el tiempo de mejoría estimado sin uso de PRP(11,12). Estos aspectos son cruciales en deportistas de alto rendimiento en quienes el tiempo de reintegro a sus actividades es fundamental.

Este tratamiento está indicado esencialmente, en los desgarros musculares fasciculares y otros de menor tamaño, pero con estricta indicación del médico tratante ${ }^{(17)}$.

La técnica consiste en la aspiración del hematoma local y su posterior infiltración con PRP en un volumen que debe ser similar o menor a aquel aspirado. Las longitudes de las agujas variará en función de la profundidad de la lesión. 

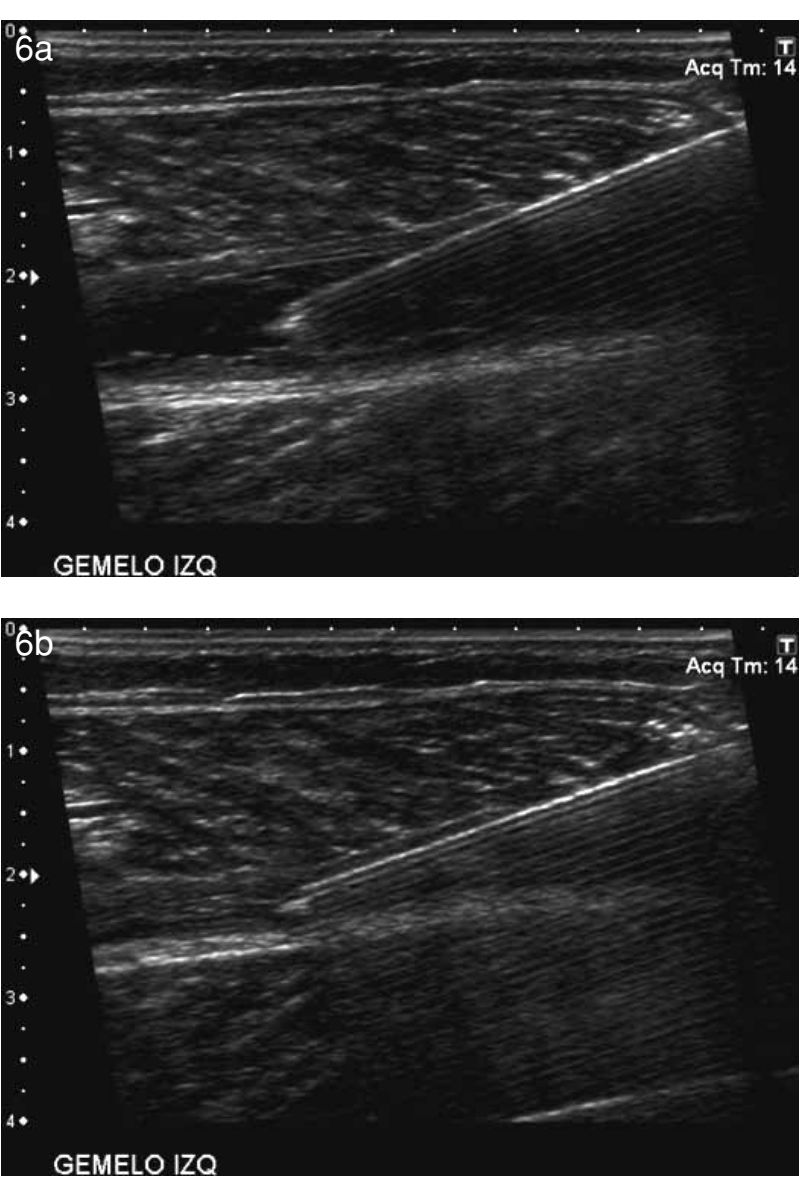

Figura 6. Desgarro muscular gemelo interno izquierdo: Mediante técnica guiada por us se procede a aspiración de hematoma y posterior infiltración de prp.

\section{Conclusiones}

El ultrasonido representa una técnica diagnóstica ampliamente extendida en el estudio y diagnóstico de la patología musculoesquelética y últimamente se ha valorizado como una herramienta altamente eficaz para guiar procedimientos intervencionales mínimamente invasivos. Debe enfatizarse que se requiere de un operador experimentado que asegure el éxito del procedimiento y disminuya la posibilidad de complicaciones. Su uso se extiende al manejo terapéutico de formaciones quísticas, infiltración de bursas y aspiración de calcificaciones intratendíneas, así como también tiene un rol coadyuvante terapéutico en diversos tipos de tendinopatías y lesiones musculares de la práctica deportiva, a través de la inyección de factores estimuladores de la regeneración y cicatrización. El imaginólogo a cargo del procedimiento debe estar familiarizado con la patología a tratar, de la anatomía regional y del arsenal farmacológico a utilizar para lograr el máximo beneficio clínico posible.

\section{Bibliografía}

1. Cardinal E, Chhem RK, Beauregard CG. UltrasoundGuided Interventional Procedures In The Musculoskeletal System. Radiol Clin North Am 1998; 36: 597-604.

2. Adler RS, Sofka CM. Percutaneous Ultrasound-Guided Injection in the Musculoskeletal System. Ultrasound Q 2003; 19(1): 3-12.

3. O'Dwyer HM, Lyon SM, Fotheringham T, Lee MJ. Informed Consent for Interventional Radiology Procedures: a Survey Detailing Current European. Cardiovasc Intervent Radiol 2003; 26(5): 428-433.

4. Louis LJ. Musculoskeletal Ultrasound Intervention: Principles and Advances. Radiol Clin North Am 2008; 46: 515-533 Practice.

5. Zubowicz VN, Ishii $\mathrm{CH}$. Management of Ganglion Cyst of the Hand by Simple Aspiration. J Hand Surg (AM). 1987; 12(4): 618-620.

6. De Smet AA. Ultrasound Guided Injections and Aspirations of the Extremities. Semin Roentgenol 2004; 39: 145-154.

7. Chiou HJ et al. Alternative and Effective treatment of Shoulder Ganglion Cyst: Ultrasonographically Guided Aspiration. J Ultrasound Med 1999; 18: 531-535.

8. Lin J, Jacobson JA, David PF, William JW, Curtis WH. An Illustrated Tutorial of Musculoskeletal Sonography: Part 4, Musculoskeletal Masses, Sonographically Guided Inteventions, and Miscellaneous Topics. AJR 2000; 175: 1711-1719.

9. Peetrons P et al. Inteventional Procedures in Musculoskeletal Ultrasound. Seminars in Inteventional Radiology 2002; 19: 189-196.

10. Farin PU, Rasanen $\mathrm{H}$, Jaroma $\mathrm{H}$ et al. Rotator Cuff Calcifications: Treatment with Ultrasound-Guided Percutaneous Needle Aspiration and Lavage. Skeletal Radiol 1996; 25(6): 551-554.

11. Aina R, Cardinal E, Bureau NJ et al. Calcific Shoulder Tendinitis; Treatment with Modified US-Guided Fine Needle Technique. Radiology 2001; 221(2): 455-461.

12. Farin PU, Jaroma H, Soimakallio. Rotator Cuff Calcifications: Treatment with US-Guided Technique. Radiology 1995; 195 (3): 841-843.

13. Speed CA. Fortnightly Review: Corticoid Injection in Tendon Lesions. BMJ 2001; 323(7309): 382-386.

14. Schiavone Panni A, Tartarone M, Maffulli N. Patellar Tendinopathy in Athletes. Outcome of Nonoperative and Operative Management. Am J Sports Med 2000; 28(3): 392-397.

15. Mishra A, Woodall J, Vieira A. Treatment of Tendon and Muscle Using Platelet-Rich Plasma. Clin Sports Med 2009; 28: 113-125.

16. Lee KS, Wilson JJ, Rabago DP, Baer GS, Jacobson JA, Borrero CG. Musculoskeletal Applications of Platelet-Rich Plasma: Fad or Future? AJR 2011; 196: 628-636.

17. Verdugo MA. Clasificación Ultrasonográfica de los Desgarros Musculares. Rev Chil Radiol 2004; 10(2): 53-57. 